\title{
Three-dimensional static displacement, stimulated echo NMR elasticity imaging
}

\author{
Derek D Steele $\dagger$, Thomas L Chenevert $\$$, Andrei R Skovoroda\| and \\ Stanislav Y Emelianov $\dagger \|$ \\ $\dagger$ Department of Biomedical Engineering, University of Michigan Medical Center, \\ 3310 Kresge III, Ann Arbor, MI 48109-0553, USA \\ $\ddagger$ Department of Radiology, University of Michigan Hospitals, 1500 E. Medical Center Drive, \\ Ann Arbor, MI 48109-0030, USA \\ || The Institute of Mathematical Problems of Biology, Russian Academy of Sciences, Pushchino, \\ Russia 142292
}

Received 16 August 1999

\begin{abstract}
This article presents a method for measuring three-dimensional mechanical displacement and strain fields using stimulated echo MRI. Additional gradient pulses encode internal displacements in response to an externally applied deformation. By limiting the mechanical transition to the stimulated echo mixing time, a more accurate static displacement measurement is obtained. A three-dimensional elasticity reconstruction within a region of interest having a uniform shear modulus along its boundary is performed by numerically solving discretized elasticity equilibrium equations. Data acquisition, strain measurements and reconstruction were performed using a silicone gel phantom containing an inclusion of known elastic properties. A comparison between two-dimensional and three-dimensional reconstructions from simulated and experimental displacement data shows higher accuracy from the three-dimensional reconstruction. The longterm objective of this work is to provide a method for remotely palpating and elastically quantitating manually inaccessible tissues.
\end{abstract}

\section{Introduction}

\subsection{Motivation}

Palpation has long been used by physicians as a means to detect disease. The underlying basis for this detection is the presence of 'hard' tissue. Evidence suggests that Young's (or shear) elastic moduli may differ by orders of magnitude within soft tissues in various physiological states (Sarvazyan et al 1995, Skovoroda et al 1995b). In addition, manual self-examination is the first diagnostic line of defence against both breast (Hill et al 1988, Newcomb et al 1991) and testicular cancers. With breast cancer, manual detection of a new mass often merits excisional biopsy, even if uncorroborated by other tests, as nodule hardness raises suspicion of malignancy (Foster 1996). Palpation of superficial lymph nodes and abdominal organs is also routinely performed. Although the touch of a skilled physician is a powerful diagnostic tool, palpation sensitivity is relatively poor within deep, dense or heterogeneous tissue. Thus, most manually detected lesions are either superficial, relatively large or both.

$\S$ Author to whom correspondence should be addressed. 


\subsection{Elasticity imaging}

Currently, many scientists are working on extending the range and sensitivity of palpation by using various methods to image tissue elasticity. The basic method for creating an elasticity map involves two steps. First, the internal displacements within tissue under an applied mechanical stress are measured. The (usually externally) applied deformation may be either dynamic or static. Then, from these data, a reconstruction of regional variations in tissue elasticity is performed, either directly or after calculating internal strains. Although both internal displacements and strains are related to the elastic properties of tissue, they are also strongly affected by geometry. Thus, some form of reconstruction is necessary to uniquely determine the elasticity distribution.

To date, two major medical imaging modalities have been used to measure tissue displacement: ultrasound and magnetic resonance imaging (MRI). The phase sensitivity of these methods lends itself to tracking tissue motion. Most elasticity imaging has been carried out using ultrasonically measured tissue displacements. These data have been obtained by tracking specular reflections (Dickinson and Hill 1982, Tristam et al 1986, 1988), by Doppler techniques (Lerner et al 1990, Parker et al 1990, Parker and Lerner 1992), by cross-correlation of acoustic echoes (Ophir et al 1991, Garra et al 1997) and by speckle tracking (Adler et al 1989, O’Donnell et al 1994, Emelianov et al 1995). Other efforts employ MRI for measuring tissue motion, as discussed below.

\subsection{MRI measurement of tissue displacement}

In the past, myocardial motion and strain have been measured using spatial magnetization tagging (Axel and Dougherty 1989, Zerhouni et al 1988), and phase-based velocity encoding (Pelc et al 1995). More recently, methods have been devised to measure tissue displacement specifically for elasticity imaging. These measurements can be separated based upon the nature of the applied deformation.

1.3.1. Dynamic deformation. With these methods, a periodic excitation is applied to the tissue near the region of interest, and the entire system may be allowed to reach steady state. One or several 'snapshots' of mechanical wave propagation within the object are produced by controlling the relative phase between the mechanical excitation and the motion-encoding gradients. The local displacement information in these images is then used as an input for an elasticity reconstruction algorithm. Initial experiments used a shear excitation, and the elasticity reconstruction was performed assuming the recorded image contained only shear waves (Muthupillai et al 1995). If only shear waves are present in a purely elastic medium, local elastic modulus variations are determined via the relation $\mu=v^{2} \lambda^{2} \rho$, where $\mu$ is the local shear modulus, $v$ is the frequency of the applied deformation, $\lambda$ is the measured local strain-wave wavelength and $\rho$ is the density of the medium. Although attractive in its simplicity, this approach is compromised by frequency-dependent viscoelastic effects and strain-wave wavelength, interference from reflections off of elastic inhomogeneities and the possible presence of longitudinal mechanical waves in the medium. Despite these limitations, this method has been applied in vivo (Dresner et al 1999, Lawrence et al 1999). Recently, a more general elasticity reconstruction from a series of 'instantaneous' steady state mechanical wave images has been developed (Sinkus et al 1999, 2000). This and another technique (Van Houten et al 1999, Weaver et al 1999) rely on a more complete viscoelastic tissue model than that presented in Muthupillai et al (1995). 
1.3.2. Static deformation. Another method of producing an internal strain field in an object is to deform it and allow the material to relax to equilibrium before measuring the displacement field. The displacement field has been accessed using spatial magnetization tagging, but this method suffers from spatial resolution limited by the tagged grid size and typically measures only two-dimensional (2D) motion (Fowlkes et al 1995). A quasistatic method using bipolar gradient phase encoding of 2D motion is presented by Plewes et al $(1995,1996)$. Stimulated echo MRI has also been used to measure 2D displacement fields (Reese et al 1996), from which elasticity images have been reconstructed (Chenevert et al 1998). This method has been extended to study myocardial motion (Aletras et al 1999b). With these techniques, viscoelastic effects are generally ignored, making the reconstruction more straightforward. Care must be taken, however, to justify the use of a static model, especially when repeated deformations are needed to acquire a complete data set.

In general, MRI has several advantages over ultrasound with respect to elasticity imaging. Although ultrasound accurately measures motion along the beam axis, lateral motion is measured with a resolution given by the depth-dependent beam width. Out-of-plane motion is generally not considered, given the problems with three-dimensional (3D) image registration in ultrasound. These restrictions compromise the quality of displacement data available and constrain the type of model used to produce an elasticity image. Ultrasound does, though, offer the advantages of low-cost and real-time imaging. MRI, on the other hand, gives one the ability to measure 3D displacements within an object, and does this at a higher overall resolution than clinical ultrasound.

In this paper we present a method for encoding the full 3D displacement field within an object undergoing an externally applied static (or quasistatic) deformation. Local strain estimates are calculated from the measured displacements, and the strain tensor is used to numerically solve differential elasticity equilibrium equations, ultimately producing a 3D elasticity image.

\section{Reconstructive elasticity imaging from static displacement fields}

The goal of elasticity imaging is to produce a map of the tissue elastic modulus in a region of interest using available measurements of displacement components. In this work, the reconstruction approach taken is based upon a model of linear, elastic, isotropic media (Skovoroda et al 1995a, 1999). The central equations and concepts are covered briefly here. A more detailed discussion can be found in the references mentioned. Note that some tissues, such as skeletal muscle, exhibit anisotropic elasticity (Fung 1993). For anisotropic media, a more generalized reconstruction method is needed.

\subsection{Linear elasticity and reconstruction}

In linear elasticity, the components of the strain $\left(\varepsilon_{i j}\right)$ and stress $\left(\sigma_{i j}\right)$ tensors in a medium undergoing small deformations are given by

$$
\begin{aligned}
\varepsilon_{i j} & =\frac{1}{2}\left(\frac{\partial u_{i}}{\partial x_{j}}+\frac{\partial u_{j}}{\partial x_{i}}\right) \\
\sigma_{i j} & =p \delta_{i j}+2 \mu \varepsilon_{i j}
\end{aligned}
$$

where $u_{i}$ is a component of the displacement vector $\boldsymbol{U}=\left(u_{1}, u_{2}, u_{3}\right)$ in Cartesian coordinates $\boldsymbol{r}=\left(x_{1}, x_{2}, x_{3}\right), p$ is the product $\lambda \nabla \cdot \boldsymbol{U}$ for compressible media or the static internal pressure for incompressible media, $\delta_{i j}$ is the Kronecker delta function, $\lambda$ and $\mu$ are the Lamé coefficients and $\mu=\mu(\boldsymbol{r})$ is the shear elastic modulus. 
A medium undergoing static deformation obeys the equilibrium condition:

$$
\sum_{j=1}^{3} \frac{\partial \sigma_{i j}}{\partial x_{j}}+f_{i}=0 \quad i=1,2,3
$$

where $f_{i}$ is the body force per unit volume acting in the $x_{i}$ direction. In addition, if a medium is incompressible, volume conservation leads to the following relation:

$$
\nabla \cdot \boldsymbol{U}=\varepsilon_{11}+\varepsilon_{22}+\varepsilon_{33}=\frac{\partial u_{1}}{\partial x_{1}}+\frac{\partial u_{2}}{\partial x_{2}}+\frac{\partial u_{3}}{\partial x_{3}}=0 .
$$

Although not necessary in the development that follows, soft tissue is approximately incompressible (Sarvazyan et al 1995).

Using equations (1) and (2) in (3), the unknown $p(\boldsymbol{r})$ can be eliminated to yield a set of differential equations depending only on $\boldsymbol{U}$, first- and higher-order spatial derivatives of $\boldsymbol{U}$, and the elasticity distribution, $\mu(\boldsymbol{r})$. This set of equations is then numerically solved to estimate the unknown shear elasticity distribution.

\subsection{Importance of three-dimensional reconstruction methods}

Several approaches have been proposed to estimate tissue elasticity from the experimentally measured spatial distribution of internal displacements within an object. The simplest method is a one-dimensional (1D) estimation of normalized tissue elasticity, expressed as

$$
\kappa_{1}=1 / \varepsilon
$$

where $\varepsilon$ is longitudinal strain (Ophir et al 1991, Garra et al 1997). Indeed, a loaded object generally exhibits low longitudinal strain in relatively hard regions and high longitudinal strain in relatively soft regions.

A 2D elasticity reconstruction, based on a plane-strain assumption and all necessary inplane strain components, provides a more accurate representation of the object's elasticity (Skovoroda et al 1995a, 1999). The theory of reconstructing clearly bounded and spatially distributed tissue inhomogeneities has been demonstrated by Skovoroda et al (1995a) as well. However, inaccurate estimates may result by using either a $1 \mathrm{D}$ or $2 \mathrm{D}$ reconstruction of a 3D object.

To demonstrate these inaccuracies, consider a spherical inclusion of radius $R$ in a uniaxially, uniformly loaded, infinite, homogeneous medium (Goudier 1933). For an incompressible medium, the distribution of longitudinal strain along the $x_{3}$ axis (orthogonal to the applied deformation), is (Skovoroda et al 1994):

$$
\varepsilon= \begin{cases}\frac{5 \beta}{3+2 \kappa_{0}} & x_{3} \leqslant R \\ \beta\left\{1+\frac{\kappa_{0}-1}{2\left(3+2 \kappa_{0}\right)}\left[5\left(\frac{R}{x_{3}}\right)^{3}-9\left(\frac{R}{x_{3}}\right)^{5}\right]\right\} & x_{3}>R .\end{cases}
$$

Here $\beta$ is the magnitude of the applied strain and $\kappa_{0}=\mu / \mu_{0}$ is the ratio of the inclusion to background shear moduli. Normalizing (6) by $\beta$, which corresponds to the axial strain in the tissue far from the inclusion, and substituting into (5) we obtain

$\kappa_{1}= \begin{cases}\frac{3+2 \kappa_{0}}{5} & x_{3} \leqslant R \\ \left(3+2 \kappa_{0}\right)\left\{3+2 \kappa_{0}+\frac{\kappa_{0}-1}{2}\left[5\left(\frac{R}{x_{3}}\right)^{3}-9\left(\frac{R}{x_{3}}\right)^{5}\right]\right\}^{-1} & x_{3}>R .\end{cases}$

Note that $\kappa_{1} / \kappa_{0}=\left(3+2 \kappa_{0}\right) / 5 \kappa_{0}$ within the inclusion. That is, for a very hard inclusion ( $\kappa_{0}$ large), the relative modulus obtained from a 1D reconstruction will only be $40 \%$ of 
(a) $\kappa\left(x_{1}, x_{2}\right)$

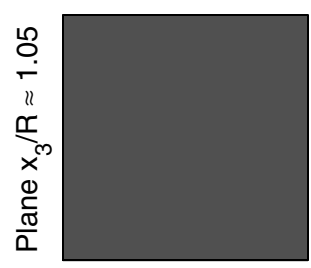

(e) $\kappa\left(x_{1}, x_{2}\right)$

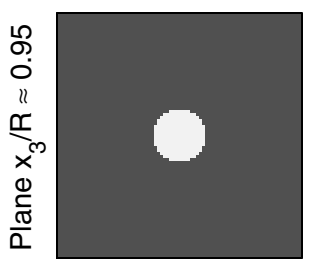

(b) $\kappa_{2}\left(x_{1}, x_{2}\right)$

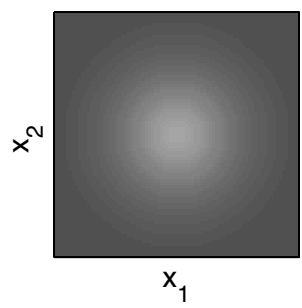

(f) $\kappa_{2}\left(x_{1}, x_{2}\right)$

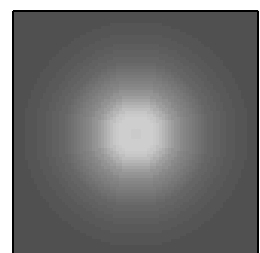

(c) $\kappa_{3}\left(x_{1}, x_{2}\right)$

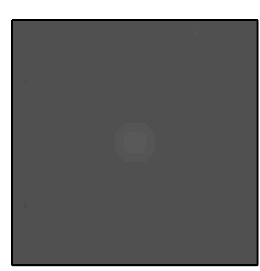

(g) $\kappa_{3}\left(x_{1}, x_{2}\right)$

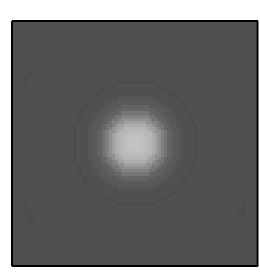

(d) Central profiles

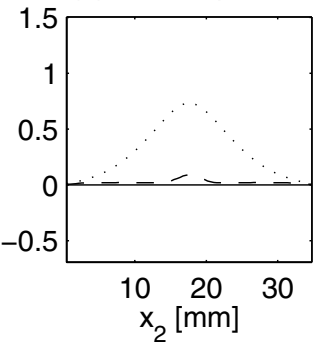

(h) Central profiles

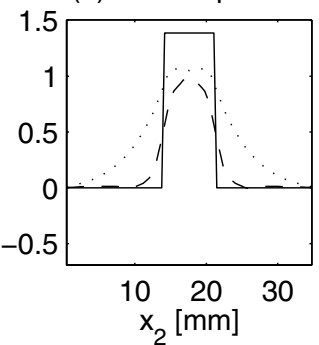

Figure 1. Simulated elasticity distributions, $\kappa\left(x_{1}, x_{2}\right)$, and corresponding $2 \mathrm{D}, \kappa_{2}\left(x_{1}, x_{2}\right)$ and $3 \mathrm{D}$, $\kappa_{3}\left(x_{1}, x_{2}\right)$, elasticity reconstructions from the $x_{3} / R \approx 1.05,(a)-(d)$, and $x_{3} / R \approx 0.95,(e)-(h)$, planes of a phantom with a single hard, spherical inclusion of radius $R$. Also presented are the central vertical profiles of each distribution, where $(-)$ is $\kappa,(\ldots \ldots)$ is $\kappa_{2}$, and $\left.(--)^{-}\right)$is $\kappa_{3}$. All are presented on a log scale where black corresponds to a relative shear modulus of 0.5 and white to 4.5 . The background has a relative shear modulus of 1 , and the inclusion, 4 .

its actual value. On the other hand, for a soft inclusion $\left(\kappa_{0}\right.$ small), the relative modulus estimate will approach $\frac{3}{5}$, no matter how much softer the inclusion is than the background. Obviously, the inaccuracy of a 1D elasticity estimation may not be acceptable for many applications.

Now consider a 2D reconstruction. Figures $1(a)$ and $(e)$ show the exact relative elasticity distribution, $\kappa\left(x_{1}, x_{2}\right)$, for two infinitesimal planes in our pedagogic phantom with $\kappa_{0}=4$. Figure $1(a)$ presents $\kappa$ for $x_{3} / R \approx 1.05$, that is, outside of the inclusion, while figure $1(e)$ is the $x_{3} / R \approx 0.95$ plane. The corresponding relative $2 \mathrm{D}$ reconstructions, $\kappa_{2}\left(x_{1}, x_{2}\right)$, are shown in figures $1(b)$ and $(f)$. The reconstructions were performed using the algorithm presented by Skovoroda et al (1999). For comparison with experimental results (see section 5), an analytic model was used to generate displacement data which were sampled with the $x_{2}$ resolution of the experimental displacement encoded data discussed in section 4.2. The strains used as input for the reconstructions were calculated as described in section 4.3 , and the reconstructions were performed over a region of interest identical to the one discussed in that same section. The positions of the two reconstructed planes were selected to approximately correspond to the experimental planes considered in section 5. As evidenced here, neglecting out-of-plane strain components in the reconstruction produces geometrical distortions in the elasticity image. Specifically, the spherical inclusion is reconstructed as a prolate spheroid. The inaccuracy of a plane-strain based reconstruction is small near the central plane, and increases with the distance between the imaging plane and the centre of the inclusion. Far from the inclusion, a $2 \mathrm{D}$ reconstruction would again be accurate. 
It is clear that a $1 \mathrm{D}$ or $2 \mathrm{D}$ reconstruction may lead to significant inaccuracies in tissue elasticity estimations, especially when complicated in vivo geometries influence displacement and strain measurements. This points to the need for an accurate $3 \mathrm{D}$ elasticity reconstruction. A general unknown shear elasticity distribution, $\mu\left(x_{1}, x_{2}, x_{3}\right)$, must satisfy the equation (Skovoroda et al 1995a, 1999):

$$
\frac{\partial^{2}\left(\mu \varepsilon_{12}\right)}{\partial x_{1}^{2}}-\frac{\partial^{2}\left(\mu \varepsilon_{12}\right)}{\partial x_{2}^{2}}+\frac{\partial^{2}\left[\mu\left(\varepsilon_{22}-\varepsilon_{11}\right)\right]}{\partial x_{1} \partial x_{2}}+\frac{\partial^{2}\left(\mu \varepsilon_{23}\right)}{\partial x_{1} \partial x_{3}}-\frac{\partial^{2}\left(\mu \varepsilon_{13}\right)}{\partial x_{2} \partial x_{3}}=0 .
$$

Thus, in order to compute all the necessary components of the strain tensor, $\varepsilon_{i j}$, in (8), all of the displacement components $\left(u_{1}, u_{2}, u_{3}\right)$ must be measured as a function of spatial coordinates $\left(x_{1}, x_{2}, x_{3}\right)$. This requirement exists in both the differential-based 3D reconstruction (8), as well as in the more stable integral based 3D approach (Skovoroda et al 1999).

The 3D elasticity reconstructions from the two planes previously discussed are shown in figures $1(c)$ and $(g)$. The reconstruction was performed as discussed in section 4.3. Although not perfect due to the relatively large $x_{3}$ step size, the $3 \mathrm{D}$ reconstructions clearly exhibit fewer geometric distortions than the 2D estimates. This is particularly well illustrated by the central vertical profiles through the analytic, 2D, and 3D shear distributions presented in figures $1(d)$ and $(h)$. In the $x_{3} / R \approx 1.05$ plane, the $2 \mathrm{D}$ reconstruction estimates that an inclusion is present, when indeed it is not, while the $3 \mathrm{D}$ reconstruction shows little evidence of the presence of an inclusion. The estimate of the extent of the inclusion in the $x_{3} / R \approx 0.95$ plane is also improved over the $2 \mathrm{D}$ estimate. As with the $2 \mathrm{D}$ reconstructions, the strain data and reconstruction parameters used for the $3 \mathrm{D}$ reconstruction were identical to those of the experimental parameters described in sections $4.2,4.3$ and 5.

\section{Static displacement measurement via stimulated echo MRI}

Static displacement measurements for elasticity imaging avoid several confounding factors that may be present if dynamic displacement measurements are used. Since shear wave propagation speed in soft tissue is approximately $1-20 \mathrm{~m} \mathrm{~s}^{-1}$, shear waves launched into a medium by an applied deformation may require tens of milliseconds to traverse an object approximately $100 \mathrm{~mm}$ in size. Reflected waves may take much longer to dampen. To appropriately measure an object's internal static displacements, the object must be in mechanical equilibrium-that is, it must satisfy (3) - during both the pre- and post-deformation measurements. A stimulated echo MRI sequence using displacement encoding gradient pulses is employed to achieve this (Reese et al 1996, Chenevert et al 1998). Figure 2 presents a schematic of this pulse sequence. The mechanical transition from the pre- to post-deformational states occurs during the stimulated echo mixing time, $T_{\mathrm{M}}$. Because the relevant magnetization is longitudinal during $T_{\mathrm{M}}$, it is unaffected by the object's motion during the mechanical transition period. This allows a more accurate measurement of static internal displacement. Additionally, precise synchronization of the motion and applied gradients is not necessary as long as the mechanical deformation begins after the second radio-frequency pulse, and internal motion stops before the third. A long delay in the echo time, $T_{\mathrm{E}}$, could also be used to let the object reach equilibrium, but this would likely lead to prohibitive signal loss from $T_{2}$ decay.

Local displacements are encoded in the magnetization's phase via pulsed-field gradients. The displacement sensitivity, in radians/distance, of the sequence is

$$
\boldsymbol{\Phi}_{\mathrm{d}}=\gamma \int_{0}^{\tau} \boldsymbol{G}_{\mathrm{d}}(t) \mathrm{d} t=\gamma \boldsymbol{G}_{\mathrm{d}} \tau
$$

where $\gamma$ is the gyromagnetic ratio of the proton, $\boldsymbol{G}_{\mathrm{d}}(t)$ is the encoding gradient waveform and $\tau$ is the duration of the encoding gradient. However, for accurate displacement measurements, 


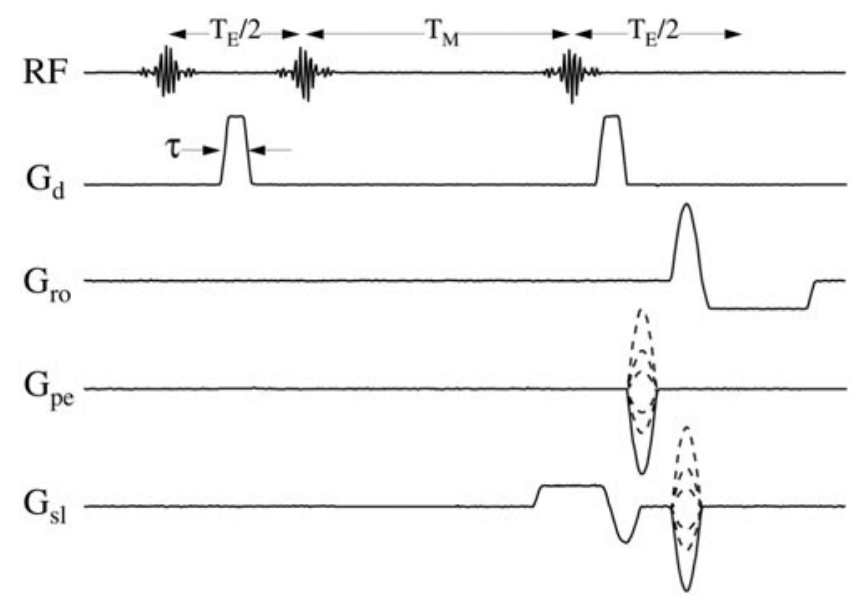

Figure 2. Displacement encoding, stimulated echo pulse sequence waveforms. $R F=$ radio frequency, $G_{\mathrm{d}}=$ displacement encoding gradient, and $G_{\text {ro }}=\operatorname{read}$-out $\left(x_{1}\right), G_{\mathrm{pe}}=$ phase-encode $\left(x_{2}\right)$ and $G_{\mathrm{sl}}=$ slice $\left(x_{3}\right)$ directed gradient waveforms. $T_{\mathrm{M}}$ is the mixing time, $T_{\mathrm{E}}$ is the echo time and $\tau$ is the duration of the displacement encoding gradient. Note that the displacement encoding gradient may be applied to any of the directional waveforms.

phaseshifts unrelated to the applied deformation must be removed. This is done by acquiring a phase reference data set using the same pulse sequence, but with the object maintained in the post-deformational state for the entire experiment. Note that all spatial encoding takes place with the object in the post-deformational state for both the displacement encoded and reference acquisitions. Therefore no image registration or tracking algorithms are required to use the reference data, $S_{\mathrm{r}}$, to correct the displacement encoded data, $S_{\mathrm{d}}$. The corrected data set, $S_{\mathrm{c}}$, is then:

$$
S_{\mathrm{c}}(\boldsymbol{r})=\frac{S_{\mathrm{d}}(\boldsymbol{r}) S_{\mathrm{r}}(\boldsymbol{r})^{*}}{\left|S_{\mathrm{r}}(\boldsymbol{r})\right|} \approx\left|S_{\mathrm{d}}(\boldsymbol{r})\right| \mathrm{e}^{\mathrm{i} \phi(r)} .
$$

Most sources of phase error, such as static field inhomogeneities, tend to be slowly varying functions of position. Thus the phase reference data may be acquired at relatively low spatial resolution to reduce scan time.

The unwrapped phase of (10) is related to the local displacement vector, $\boldsymbol{U}$, via

$$
\phi(r)=\Phi_{\mathrm{d}} \cdot \Delta \boldsymbol{r}=\Phi_{\mathrm{d}} \cdot \boldsymbol{U}(\boldsymbol{r})
$$

where $\Delta r$ is the local displacement from pre- to post-deformational states. The displacement sensitivity, $\boldsymbol{\Phi}_{\mathrm{d}}$, may be made sensitive to motion in an arbitrary direction based upon appropriate combination of displacement encoding gradients in the read-out, phase-encode and slice directions. Hence, this pulse sequence readily extends to acquiring three-dimensional displacement data.

\section{Methods}

\subsection{Phantom}

Elasticity imaging experiments were performed on a phantom with a spherical hard inclusion. Semicosil 921 silicone gel (Wacker Silicones Corporation, Adrian, MI) was used to construct a phantom qualitatively simulating the mechanical properties of soft tissue. The Semicosil 921 
consists of two components, $\mathrm{A}$ and $\mathrm{B}$, wherein different ratios of these components are used to vary the mechanical properties of the gel. A tissue-mimicking phantom was constructed in several steps. First, background material was prepared by thoroughly mixing components $A$ and $B$ in a 1:1 ratio, and then pouring the mixture into a $154 \mathrm{~mm}$ by $80 \mathrm{~mm}$ rectangular mould. The mixture was degassed and cured for $24 \mathrm{~h}$ at room temperature to produce a $22 \mathrm{~mm}$ thick layer. Then a $25 \mathrm{~mm}$ diameter hard sphere was prepared from a 1:2.5 mixture of $\mathrm{A}$ and $\mathrm{B}$ and was placed on top of the layer in the middle of the mould. Finally, another batch of background material (1:1 ratio) was poured into the mould resulting in a $64 \mathrm{~mm}$ by $80 \mathrm{~mm}$ by $154 \mathrm{~mm}$ phantom with a single, hard, spherical inclusion roughly in the centre. At the same time, three samples of each batch were taken to independently assess the elasticity contrast between the inclusion and surrounding materials. These measurements were performed using the force-deformation system described in Erkamp et al (1998), and showed that the inclusion was four times harder than the background, and that both background materials were elastically equivalent.

\subsection{Data acquisition}

To provide repeatable deformation, the phantom was placed under moderate pre-load pressure between two acrylic plates in a pneumatically driven device. Air-filled neoprene boots in a push-push configuration provided the necessary force to the top plate to keep the phantom in this pre-load state, and aided the vertical recoil of the phantom to the post-deformation state. Pneumatic pressure was delivered via two solenoid valves whose timing was controlled by an external transistor-transistor logic circuit triggered by the pulse sequence. Quick-release valves aided in depressurizing the boots. Both the pre-load and recoil positions of the top acrylic plate were set by adjustable stops; the bottom plate's position was fixed. The applied vertical deformation was approximately $2.4 \mathrm{~mm}$, or about $6 \%$ strain, between the pre-transition (greater deformation) and post-transition (less deformation) states.

During data acquisition, the displacement encoding gradient pulse duration, $\tau$, was $1.5 \mathrm{~ms}$, and the amplitude, $G_{\mathrm{d}}$, was $40 \mathrm{mT} \mathrm{m}^{-1}$ in the read-out $\left(x_{1}\right)$ and phase-encode $\left(x_{2}\right)$ directions, and $60 \mathrm{mT} \mathrm{m}^{-1}$ in the slice $\left(x_{3}\right)$ direction. Here, the $x_{3}$ direction was along the bore's axis, and the $x_{1}$ and $x_{2}$ directions were perpendicular to $x_{3}$ in the horizontal and vertical directions respectively. By (9), the displacement sensitivity, $\Phi_{\mathrm{d}}$, was approximately $5.11 \pi \mathrm{mm}^{-1}$ in the $x_{1}$ and $x_{2}$ directions, and about $7.66 \pi \mathrm{mm}^{-1}$ in the $x_{3}$ direction. The displacement encoding direction was cycled each pulse repetition between the $x_{1}, x_{2}$ and $x_{3}$ directions. The pulseto-pulse repetition time was approximately $0.98 \mathrm{~s}$, the mixing time $\left(T_{\mathrm{M}}\right)$ was $270 \mathrm{~ms}$, and the echo time $\left(T_{\mathrm{E}}\right)$ was $45 \mathrm{~ms}$. Two averages were taken of a $256 \times 256 \times 32$ matrix covering an $80 \mathrm{~mm}$ by $110 \mathrm{~mm}$ by $48 \mathrm{~mm}$ field of view. The phase reference data were collected using a $256 \times 32 \times 32$ matrix while keeping all other parameters the same. All experiments were performed on a $2 \mathrm{~T}, 18 \mathrm{~cm}$ bore MRI system (Bruker, formerly GE NMR Instruments) using a $150 \mathrm{~mm}$ transmit/receive birdcage coil.

\subsection{Data processing}

All time-domain data were transferred off-line for processing. For phase correction, the phase reference data set was zero-filled to a $256 \times 256 \times 32$ matrix. Then this and the displacement encoded data were $3 \mathrm{D}$ Fourier transformed and corrected as in (10). The resulting phase maps were then used to estimate the spatial derivatives to compute the strains, via (1), necessary for the elasticity reconstruction. Phase unwrapping of the displacement data was not strictly required since only phase derivatives were used in the strain calculations. The displacement 
derivative at the $i$ th point in the $j$ direction was computed from the angle of the complex multiplication of the $i+1$ th point with the conjugate of the $i-1$ th point, then scaling by $1 / 2 \Phi_{\mathrm{d}}^{j}$, where $\Phi_{\mathrm{d}}^{j}$ is the magnitude of the displacement sensitivity in the $j$ direction. For convenience, the strain data were decimated to the $x_{2}$ step size in each $x_{3}$ plane in order to have equal resolution in both the $x_{1}$ and $x_{2}$ directions. The strain images were then median filtered with a $5 \times 5$ window, resulting in a slight decrease in spatial resolution. These strains were used as input for the elasticity reconstruction.

The 3D elasticity reconstruction was performed using the least-squares error minimization algorithm discussed in Skovoroda et al (1999), with a second-order, one-sided finite derivative approximation in the $x_{3}$ direction. The reconstruction of $\mu(\boldsymbol{r})$ is a boundary value problem, therefore a unique solution is obtained only with boundary conditions. So a square $35 \mathrm{~mm}$ by $35 \mathrm{~mm}$ region of interest, which contained the inclusion in several $x_{3}$ planes, was identified in the $x_{1}$ and $x_{2}$ directions. Along the boundaries of these regions, and in the two $x_{3}$ planes furthest from the centre of the inclusion (which did not contain the inclusion), the value of the shear modulus was set to 1 , resulting in a relative shear modulus reconstruction.

\section{Results}

Representative magnitude and corrected phase images of the Semicosil phantom for a $1.5 \mathrm{~mm}$ thick plane centred about $x_{3}=0.75 \mathrm{~mm}$, or $x_{3} / R \approx 0.05$, are shown in figure 3 . Knowing that $\Phi_{\mathrm{d}} \approx 5.11 \pi \mathrm{mm}^{-1}$ in the $x_{1}$ and $x_{2}$ directions, the number of $2 \pi$ phase wraps in figure $3(c)$

(a) $S_{1}\left(x_{1}, x_{2}\right)$

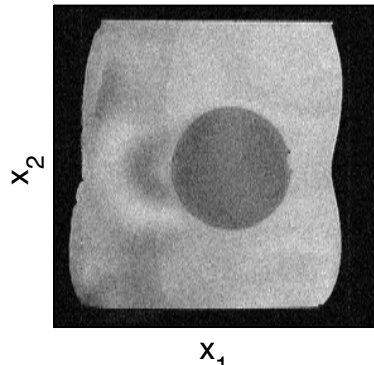

(c) $\phi_{2}\left(x_{1}, x_{2}\right)$

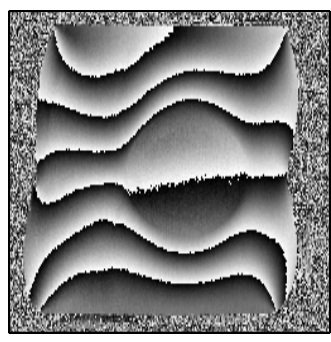

(b) $\phi_{1}\left(\mathrm{x}_{1}, \mathrm{x}_{2}\right)$

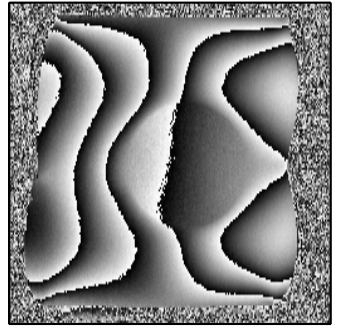

(d) $\phi_{3}\left(x_{1}, x_{2}\right)$

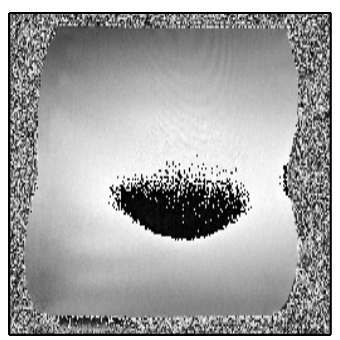

Figure 3. Representative magnitude and phase images from the $x_{3} / R \approx 0.05$ plane of the 3D displacement encoded data set from a phantom with a single, hard, spherical inclusion. $S_{1},(a)$, is the magnitude of the $x_{1}$-displacement encoded data, and $\phi_{i},(b)-(d)$, are the phase images of the $x_{i}$-displacement encoded data. 
(a) $\varepsilon_{22}\left(x_{1}, x_{2}\right)$

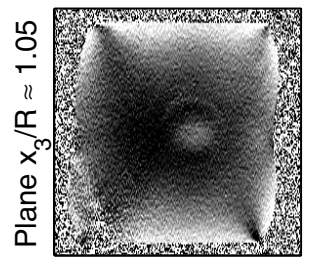

(e) $\varepsilon_{22}\left(x_{1}, x_{2}\right)$

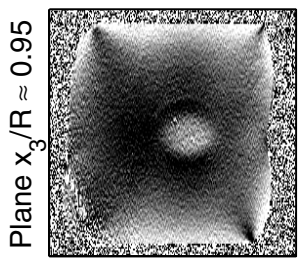

(b) $\varepsilon_{12}\left(x_{1}, x_{2}\right)$

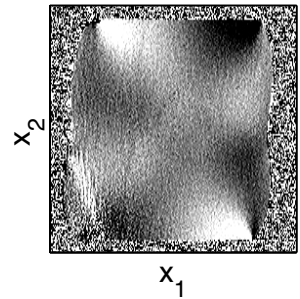

(f) $\varepsilon_{12}\left(x_{1}, x_{2}\right)$

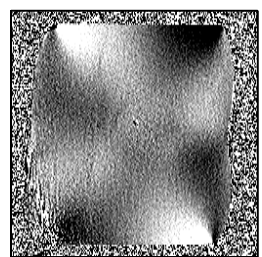

(c) $\varepsilon_{13}\left(x_{1}, x_{2}\right)$

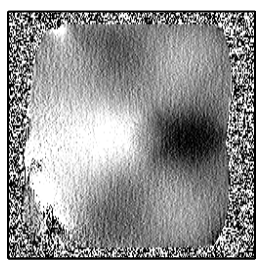

(g) $\varepsilon_{13}\left(x_{1}, x_{2}\right)$

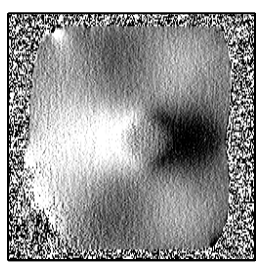

(d) $\varepsilon_{11}+\varepsilon_{22}+\varepsilon_{33}$

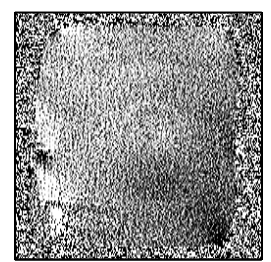

(h) $\varepsilon_{11}+\varepsilon_{22}+\varepsilon_{33}$

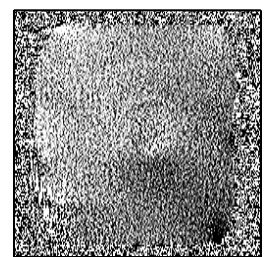

Figure 4. Representative strain images from the $x_{3} / R \approx 1.05,(a)-(d)$, and $x_{3} / R \approx 0.95,(e)-(h)$, planes from the $3 \mathrm{D}$ displacement encoded data of the phantom with a single, hard, spherical inclusion. One normal strain, $\varepsilon_{22}$, the in-plane shear strain, $\varepsilon_{12}=\varepsilon_{21}$, one through-plane shear strain, $\varepsilon_{13}=\varepsilon_{31}$, and the trace of the strain tensor, $\varepsilon_{11}+\varepsilon_{22}+\varepsilon_{33}$, are presented for each plane. The lack of features in $(d)$ and $(h)$ indicate that the phantom is nearly incompressible. Linear scales for each image are, from black to white: $(a),(e):[-6 \%, 0 \%] ;(b),(f):[-2.5 \%, 2.5 \%] ;(c),(d),(g)$, (h): $[-1.6 \%, 1.6 \%]$.

indicates a vertical deformation of approximately $2.3 \mathrm{~mm}$, and those in figure $3(b)$ a horizontal deformation of about $2.0 \mathrm{~mm}$. Reduced phase slope in the region of the hard inclusion is clearly visible in these figures as well. Due to the central location of this plane, there is little feature in $\phi_{3}$ (part $\left.(d)\right)$.

Figure 4 shows representative strain maps from the planes centred around $x_{3}=15.75 \mathrm{~mm}$ and $x_{3}=14.25 \mathrm{~mm}$. Due to the loaded state of the phantom during imaging, the sphere became prolate, therefore these planes correspond to the $x_{3} / R \approx 1.05$ and $x_{3} / R \approx 0.95$ locations respectively. One normal strain, $\varepsilon_{22}$ (parts $(a)$ and $(e)$ ), the in-plane shear strain, $\varepsilon_{12}=\varepsilon_{21}$ (parts $(b)$ and $(f)$ ), and one through-plane shear strain, $\varepsilon_{13}=\varepsilon_{31}$ (parts $(c)$ and $(g)$ ), are shown for each plane. These components are all required to perform the elasticity reconstruction in (8). Note that the presence of through-plane strains in (8) necessitates measurement of the full 3D displacement field. In addition, although elasticity-specific details are seen in the strain maps, features related to geometry and the applied deformation are also clearly present. This points to the need for a proper elasticity reconstruction to disentangle these factors. Also shown is the trace of the strain tensor, $\varepsilon_{11}+\varepsilon_{22}+\varepsilon_{33}$, for each plane (parts $(d)$ and $(h)$ ). The relative lack of features in the trace of the strain tensor indicates that the phantom is nearly incompressible (like soft tissue).

Magnitude images of the $35 \mathrm{~mm}$ by $35 \mathrm{~mm}$ regions of interest in the same two planes, along with two different shear modulus reconstructions of these planes, are presented in figure 5 . In the magnitude images, the hard inclusion is clearly present in the $x_{3} / R \approx 0.95$ plane, while it is essentially absent in the $x_{3} / R \approx 1.05$ plane. Note that the magnitude images only convey geometric information. Figures $5(b)$ and $(f)$ show 2 D elasticity reconstructions of these 
(a) $S_{1}\left(x_{1}, x_{2}\right)$

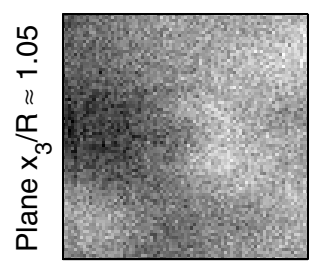

(e) $S_{1}\left(x_{1}, x_{2}\right)$

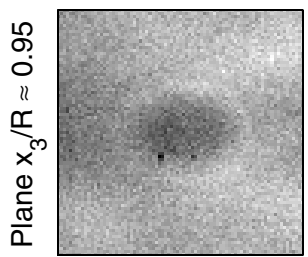

(b) $\kappa_{2}\left(x_{1}, x_{2}\right)$

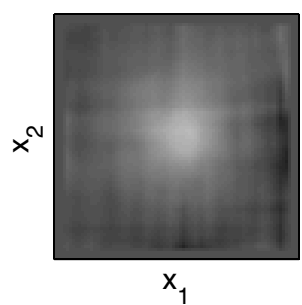

(f) $\kappa_{2}\left(x_{1}, x_{2}\right)$

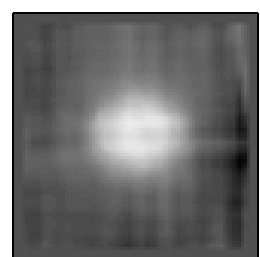

(c) $\kappa_{3}\left(x_{1}, x_{2}\right)$

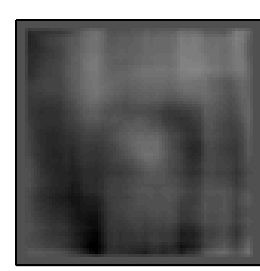

$-0.5$

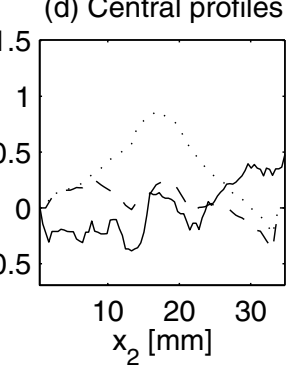

(g) $\kappa_{3}\left(x_{1}, x_{2}\right)$

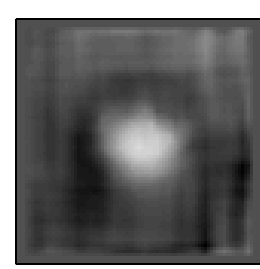

(h) Central profiles

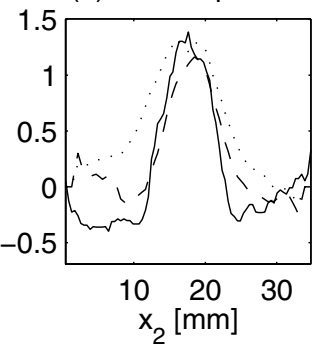

Figure 5. $x_{1}$-displacement encoded magnitude images, $S_{1}\left(x_{1}, x_{2}\right)$, and corresponding $2 \mathrm{D}$, $\kappa_{2}\left(x_{1}, x_{2}\right)$, and $3 \mathrm{D}, \kappa_{3}\left(x_{1}, x_{2}\right)$ elasticity reconstructions from the $x_{3} / R \approx 1.05,(a)-(d)$, and $x_{3} / R \approx 0.95,(e)-(h)$, planes of a phantom with a single hard, spherical inclusion. Also presented are the central vertical profiles of each distribution, where $(\ldots \ldots)$ is $\kappa_{2}$, and (- - -) is $\kappa_{3}$. All are presented on a log scale where black corresponds to a relative shear modulus of 0.5 and white to 4.5. The background has a relative shear modulus of 1 , and the inclusion, 4 , from independent measurements. For geometric reference purposes, parts $(d)$ and $(h)$ include plots of $S_{1}^{-1}$ (filtered and normalized) as ( - ).

two planes, while figures $5(c)$ and $(g)$ show the corresponding 3D elasticity reconstructions. As in figure 1, one sees an overestimate of the $3 \mathrm{D}$ spatial extent of the inclusion in the $2 \mathrm{D}$ reconstructions. This overestimate is corrected with the $3 \mathrm{D}$ reconstruction. For ease of comparison, vertical profiles through the centre of the inclusion from the $2 \mathrm{D}$ and $3 \mathrm{D}$ reconstructions are presented, along with plots of $S_{1}^{-1}$ for geometric reference, in figures $5(d)$ and $(h)$.

\section{Discussion}

The stimulated echo sequence presented here phase encodes internal displacements using gradient pulses. An externally applied deformation, synchronized with the pulse sequence, produces an internal displacement field. This deformation is actively driven with a pneumatic device, and the mechanical transition from pre- to post-deformation occurs during the sequence mixing time, $T_{\mathrm{M}}$, while the relevant magnetization is longitudinal. Because longitudinal magnetization decays only as $T_{1}$, the mechanical transition period may be extended to allow potentially long-lived or ill-defined motions within the object to dampen. With a sufficiently long $T_{\mathrm{M}}$, the encoded displacement will be approximately static. However, signal loss due to $T_{1}$ relaxation sets a practical limit on the length of $T_{\mathrm{M}}$. To determine an appropriate mixing time, a series of $2 \mathrm{D}$ displacement encoded images was taken, varying $T_{\mathrm{M}}$ from $50-750 \mathrm{~ms}$. 
From the phase maps of these data, one could see that the top acrylic plate of the deformation device completed its excursion in under $200 \mathrm{~ms}$, and the internal motion in the phantom became negligible by $250 \mathrm{~ms}$. To ensure that (3) was reasonably satisfied, a $270 \mathrm{~ms}$ mixing time was chosen for subsequent data collection. Although the deformation device used here provides adequate transition speed, an even faster device would allow a shorter $T_{\mathrm{M}}$, yielding more signal. While absent in the phantom used here, water diffusion in the presence of displacement encoding gradients will be another source of signal loss in in vivo experiments. This loss can be mitigated by reducing the displacement sensitivity, $\Phi_{\mathrm{d}}$, or by shortening $T_{\mathrm{M}}$.

The quality of the shear modulus reconstruction ultimately depends on the local phase, as in (11), induced by the encoding gradients and the local displacement. More specifically, the quality depends on the spatial derivatives of the encoded phase. A study of the effects of displacement sensitivity, applied deformation, relative hardness and diffusion loss on the signal-to-noise ratio (SNR) of the elasticity reconstruction has been presented in Steele et al (1999). This study demonstrates that increased intra-voxel phase wrap will increase the reconstruction SNR, up to a $\pi$ intra-voxel phase distribution. Note that the reconstruction SNR increases despite a reduction in the nuclear magnetic resonance (NMR) signal from the object. Assuming a linear phase distribution of $\theta$ radians across a voxel, the signal modulation from that voxel will be $|\operatorname{sinc}(\theta / 2)|=\left|\sin (\theta / 2)(\theta / 2)^{-1}\right|$. However, the phase gradients (that is, the displacement derivatives) will be maximized without aliasing as the intra-voxel phase wrap approaches $\pi$, and this is the signal that is important in the reconstruction. A $\pi$ phase wrap may be achieved through many combinations of applied deformation and displacement sensitivity. However, increasing $\Phi_{\mathrm{d}}$ will increase signal loss due to diffusion, as discussed above. Hence, a smaller displacement sensitivity and increased deformation would appear to be optimal. Again, there is a trade-off: as deformation increases, the model of linear elasticity discussed in section 2.1 will become less and less valid. Elasticity reconstructions from finite displacement fields have been demonstrated in Skovoroda et al (1999), but these are obviously more computationally intensive than the linear reconstructions used here. In relation to the data presented here, the number of $2 \pi$ phase bands across the phantom in figure 3 clearly indicate that these data were acquired with a suboptimal displacement sensitivity/applied deformation combination. Because neither the encoding nor the deformation used here were extreme, the elasticity reconstruction's SNR should be improved merely by optimizing the intra-voxel phase wrap.

Relative hardness, object geometry and deformation geometry also affect the displacement phase gradients. In general, the phase gradients increase near soft/hard interfaces and are higher in relatively soft regions of tissue. Excessive phase wrap (i.e. strain) can lead to regions of significant signal loss in a manner analogous to flow dephasing in conventional MRI. The resulting reconstructions would suffer from this signal loss. Hence, the applied deformation and displacement sensitivity should be optimized for the regions of highest strain in an object. Increased intra-voxel phase wrap in regions of lower strain may be obtained by integrating the signal from several voxels; in essence, applying an adaptive voxel size based upon local phase gradients. This increase in signal would come at the expense of spatial resolution. Additionally, signal loss due to intra-voxel phase wrap in the displacement encoded magnitude images may be useful for identifying regions of high strain in tissue.

Another factor affecting the displacement signal is the reproducibility of the applied deformation. For multistep acquisitions, such as those presented here, good deformation reproducibility is essential. Variations in the applied deformation will lead to phase instability, motion-like artefacts, and errors that will propagate through the elasticity reconstruction. Adequate reproducibility has been achieved with the current deformation system. However, irreproducible or asynchronous motions within the imaged object may be problematic. 
These would include physiological cardiac and respiratory motion present in in vivo experiments. In some ways, the problems associated with undesired motion would be similar to those encountered in diffusion MRI. Because the applied deformation is external, though, the displacement encoding can be tailored to it, reducing the effect of undesired motion on the displacement data. Further complications arise because phase derivatives of the displacement data, approximated by finite differences, are required for reconstruction. In addition to choosing an appropriate deformation/encoding combination, methods should be devised to reduce the effects of undesired motion on the displacement derivatives.

Clearly several advantages justify performing a 3D elasticity reconstruction rather than a 2D reconstruction. As illustrated in figures 1 and 5, and as discussed in sections 2.2 and 5, a 3D reconstruction provides a more accurate representation of the elasticity distribution than a $2 \mathrm{D}$ reconstruction in the simple phantom used here. Complicated in vivo geometries will only increase the likelihood that neglecting out-of-plane strain components will result in an inaccurate elasticity estimate. This increased accuracy comes, though, at the expense of increased computational complexity and increased scan time. For instance, a single acquisition of the 3D data discussed in section 4.2 takes over $6 \mathrm{~h}$ ! This far exceeds any clinically feasible scan times. The total experiment time may be lessened through the use of echo-planar imaging (Mansfield 1977) or fast spin-echoes (Hennig et al 1986) for spatial encoding (Chenevert et al 1999). A fast scan implementation has already been used to study cardiac motion (Aletras et al 1999a). The number of planes of data acquired may also be significantly reduced while still allowing a $3 \mathrm{D}$ reconstruction, shortening the scan time further. It should be mentioned that a classic 3D stimulated echo sequence was deliberately chosen in part due to SNR considerations, since a fast scan implementation of the method would generally have a lower SNR than one classically phase encoded. Being an inverse problem, the $3 \mathrm{D}$ reconstruction is sensitive to the SNR, and we wanted the initial test of the reconstruction to be done with the highest SNR data possible using this technique. Also, note that the reconstruction in (8) does not rely on the assumption of incompressibility, although making this assumption provides another means of regularizing the inverse problem.

Additionally, a reconstruction of static displacement data offers several advantages over a reconstruction of dynamic displacement data. A static reconstruction allows one to ignore viscoelastic effects as well as the longitudinal or shear nature of the applied deformation. Static methods also provide high SNR displacement and strain estimates. Dynamic methods, on the other hand, provide a potentially very simple reconstruction (Muthupillai et al 1995). However, this reconstruction may be compromised by interference from elastic inhomogeneities, attenuation of shear waves, mixing of longitudinal and shear waves, and resolution limits imposed by noise when determining the shear-wave wavelength. Reconstruction models that include viscoelastic effects allow a more accurate interpretation of dynamic data (Sinkus et al 1999, 2000, Van Houten et al 1999), but these are necessarily more complicated than static models (Skovoroda et al 1995a, 1999).

Choosing a contour of constant shear modulus for appropriate boundary conditions for (8), though, can in practice be a challenge. In the applications discussed here, a priori knowledge of phantom geometry was employed in the reconstructions. This may be possible in vivo as well, albeit more complicated. For instance, in breast elasticity imaging, such a contour may be defined in the subcutaneous fat surrounding the parenchyma using the boundary detection procedure described in Skovoroda et al (1995a). The elasticity reconstruction would then be relative to the shear modulus of the fat boundary, assuming that it is constant. Alternatively, a high signal cuff of known elastic modulus could be used to surround the breast. This would provide an absolute image of shear modulus variations if the boundary contour were chosen inside the cuff. 
The 3D shear elasticity reconstructions presented above contain artefacts both inside and outside of the inclusion due to the finite SNR in the measured displacement strain components, and due to the step size used in the finite approximation to the derivatives in the reconstruction. In contrast to the $2 \mathrm{D}$ elasticity reconstruction, where the elasticity distribution is reconstructed independently in each plane, the $3 \mathrm{D}$ reconstruction uses the elasticity distribution in neighbouring planes. Therefore, in addition to in-plane error propagation problems discussed elsewhere (Skovoroda et al 1995a), error propagation in the through-plane direction may occur due to inaccurate elasticity reconstructions in the preceding planes. This is particularly true if the 3D elasticity reconstruction is performed, as in this paper, by solving an initial value problem in the through-plane direction. Even though the more stable integral based approach (Skovoroda et al 1999) was employed to solve for $\mu(\boldsymbol{r})$ in each plane, the results of the 3D elasticity reconstructions in subsequent planes exhibit significant error propagation in the $x_{3}$ direction. Given a particular spatial discretization of the displacement data, this error propagation can be reduced by several approaches. These include more appropriate data filtering and reducing the reconstruction's sensitivity to noise, but these considerations are beyond the scope of this paper.

\section{Conclusions}

The ultimate goal of quantitative elasticity imaging is to provide physicians with a method of remotely palpating soft tissue to detect disease. The three-dimensional elasticity imaging technique demonstrated here is a step toward extending the range and sensitivity of palpation, a powerful diagnostic tool. One possible application of this technique would be measuring the elasticity of breast tissue normally inaccessible to manual palpation. A large elastic modulus difference between normal and pathological breast tissue has been measured in situ. A previous study indicates that soft tissues in different physiological states display shear modulus variations of one to two orders of magnitude (Sarvazyan et al 1995). If these elastic changes pre-date calcification formation, elasticity imaging may increase sensitivity to and characterization of malignant breast masses, complementing existing diagnostic tools. The relatively high cost of MRI may hinder using this approach as a general screening technique. However, additional work to define the role of this modality as a primary or complementary diagnostic tool in diseases of soft tissues seems worthwhile indeed.

\section{Acknowledgments}

The authors would like to thank M O'Donnell for helpful discussions and suggestions, and $\mathrm{R}$ Erkamp for the direct mechanical measurements of samples. This research was funded in part by the National Institutes of Health grant DK47324 and by US Army grant DAMD17-97-7079.

\section{References}

Adler R, Rubin J M, Bland P and Carson P 1989 Characterization of transmitted motion in fetal lung-quantitative analysis Med. Phys. $16333-7$

Aletras A H, Balaban R S and Wen H 1999a High-resolution strain analysis of the human heart with fast-DENSE J. Magn. Reson. 140 41-57

Aletras A H, Ding S J, Balaban R S and Wen H 1999b DENSE: Displacement encoding with stimulated echoes in cardiac functional MRI J. Magn. Reson. 137 247-52

Axel L and Dougherty L 1989 MR imaging of motion with spatial modulation of magnetization Radiology 171 59-63

Chenevert T L, Skovoroda A R, O’Donnell M and Emelianov S Y 1998 Elasticity reconstructive imaging by means of stimulated echo MRI Magn. Reson. Med. 39 482-90 
Chenevert T L, Steele D D, Emelianov S Y and O'Donnell M 1999 Proc. ISMRM, 7th Scientific Meeting and Exhibition (Philadelphia) p 263

Dickinson R J and Hill C R 1982 Measurement of soft-tissue motion using correlation between A-scans Ultrasound Med. Biol. 8 263-71

Dresner M A, Rossman P J, Kruse S A and Ehman R L 1999 Proc. ISMRM, 7th Scientific Meeting and Exhibition (Philadelphia) p 526

Emelianov S Y, Lubinski M A, Weitzel W F, Wiggins R C, Skovoroda A R and O’Donnell M 1995 Elasticity imaging for the early detection of renal pathology Ultrasound Med. Biol. 21 871-83

Erkamp R Q, Wiggins P, Skovoroda A R, Emelianov S Y and O'Donnell M 1998 Measuring the elastic modulus of small tissue samples Ultrason. Imaging 20 17-28

Foster R S Jr 1996 Diseases of the Breast ed J R Harris et al (Philadelphia: Lippincott-Raven) pp 133-8

Fowlkes J B, Emelianov S Y, Pipe J G, Skovoroda A R, Adler R S and Carson P L 1995 Magnetic resonance imaging techniques for detection of elasticity variation Med. Phys. 22 1771-8

Fung Y C 1993 Biomechanics: Mechanical Properties of Living Tissue (New York: Springer)

Garra B S, Cespedes E I, Ophir J, Spratt S R, Zuurbier R A, Magnant C M and Pennanen M F 1997 Elastography of breast lesions: Initial clincal results Radiology 202 79-86

Goudier J 1933 Concentration of stress around spherical and cylindrical inclusions and flaws Trans. ASME 55 39-44

Hennig J, Nauerth A and Friedburg H 1986 RARE imaging-a fast imaging method for clinical MR Magn. Reson. Med. 3 823-33

Hill D, White V, Jolley D and Mapperson K 1988 Self examination of the breast—is it beneficial—meta-analysis of studies investigating breat self examination and extent of disease in patients with breast cancer Br. J. Med. 297 271-5

Lawrence A J, Rossman P J, Mahowald J L, Manduca A, Hartmann L C and Ehman R L 1999 Proc. ISMRM, 7th Scientific Meeting and Exhibition (Philadelphia) p 525

Lerner R M, Huang S R and Parker K J 1990 Sonoelasticity images derived from ultrasound signals in mechanically vibrated tissues Ultrasound Med. Biol. 16 231-9

Mansfield P 1977 Multi-planar image formation using NMR spin echoes J. Phys. C: Solid State Phys. 10 L55-L58

Muthupillai R, Lomas D J, Rossman P J, Greenleaf J F, Manduca A and Ehman R L 1995 Magnetic resonance elastography by direct visualization of propagating acoustic strain waves Science 269 1854-7

Newcomb P A, Weiss S, Storer B E, Scholes D and Young B E 1991 Breast self-examination in relation to the occurence of advanced breast cancer J. Natl. Cancer Inst. $83260-5$

O’Donnell M, Skovoroda A R, Shapo B M and Emelianov S Y 1994 Internal displacement and strain imaging using ultrasonic speckle tracking IEEE Trans. Ultrason. Ferroelectr. Freq. Control 42 314-25

Ophir J, Cespedes E I, Ponnekanti H, Yazdi Y and Li X 1991 Elastography-a quantitative method for imaging the elasticity of biological tissues Ultrason. Imaging 13 111-34

Parker K J, Huang S R, Musulin R A and Lerner R M 1990 Tissue response to mechanical vibrations for sonoelasticity Ultrasound Med. Biol. 16 241-6

Parker K J and Lerner R M 1992 Sonoelasticity of organs—shear waves ring a bell J. Ultrasound Med. 11 387-92

Pelc N J, Drangova M, Pelc L R, Zhu Y, Noll D C, Bowman B S and Herfkens R J 1995 Tracking of cyclic motion with phase-contrast cine MR velocity data J. Magn. Reson. Imaging 5 339-45

Plewes D B, Betty I, Urchuk S N and Soutar I 1995 Visualizing tissue compliance with MR imaging J. Magn. Reson. Imaging $\mathbf{5} 733-8$

Plewes D B, Poole G, Leitch M and Urchuk S N 1996 Proc. ISMRM, 4th Scientific Meeting and Exhibition (New York) p 476

Reese T G, Wedeen V J and Weisskoff R M 1996 Measuring diffusion in the presence of material strain J. Magn. Reson. Ser. B 112 253-8

Sarvazyan A P, Skovoroda A R, Emelianov S Y, Fowlkes J B, Pipe J G, Adler R S, Buxton R B and Carson P L 1995 Acoustical Imaging vol 21 (New York: Plenum) pp 223-40

Sinkus R, Lorenzen J, Schrader D, Lorenzen M, Dargatz M and Holz D 1999 Proc. ISMRM, 7th Scientific Meeting and Exhibition (Philadelphia) p 259

-2000 High-resolution tensor MR elastography for breast tumour detection Phys. Med. Biol. 45 1649-64

Skovoroda A R, Emelianov S Y, Lubinski M A, Sarvazyan A P and O'Donnell M 1994 Theoretical analysis and verification of ultrasound displacement and strain imaging IEEE Trans. Ultrason. Ferroelectr. Freq. Control 41 $302-13$

Skovoroda A R, Emelianov S Y and O’Donnell M 1995a Tissue elasticity reconstruction based on ultrasonic displacement and strain images IEEE Trans. Ultrason. Ferroelectr. Freq. Control 42 747-65

Skovoroda A R, Klishko A N, Gukasyan D A, Maevsky E I, Ermilova V D, Oranskaya G A and Sarvazyan A P 1995 b Quantitative analysis of the mechanical characteristics of pathologically altered soft biological tissues Biofizika 40 1335-40 
Skovoroda A R, Lubinski M A, Emelianov S Y and O'Donnell M 1999 Reconstructive elasticity imaging for large deformations IEEE Trans. Ultrason. Ferroelectr. Freq. Control 46 523-35

Steele D D, Chenevert T L, Emelianov S Y and O'Donnell M 1999 Proc. ISMRM, 7th Scientific Meeting and Exhibition (Philadelphia) p 1616

Tristam M, Barbosa D C, Cosgrove D O, Bamber J C and Hill C R 1988 Ultrasonic study of in vivo kinetic characteristics of human tissues Ultrasound Med. Biol. 14 695-707

Tristam M, Barbosa D C, Cosgrove D O, Nassiri D K, Bamber J C and Hill C R 1986 Application of Fourier analysis to clinical study of patterns of tissue movement Ultrasound Med. Biol. 12 927-37

Van Houten E, Miga M I, Kennedy F E, Weaver J B and Paulsen K D 1999 Proc. ISMRM, 7th Scientific Meeting and Exhibition (Philadelphia) p 260

Weaver J B, Van Houten E, Miga M I, Kennedy F E, Hartov A, Poplack S P, Nagy H M and Paulsen K D 1999 Proc. ISMRM, 7th Scientific Meeting and Exhibition (Philadelphia) p 1617

Zerhouni E A, Parish D M, Rogers W J, Yang A and Shapiro E P 1988 Human heart: tagging with MR imaging—a method for noninvasive assessment of myocardial motion Radiology 169 164-72 